\title{
Chancen für die Adhärenz - die Patientenperspektive
}

Jeannette Saner

\author{
Laut Studien liegt die Adhärenz von Patienten für Eigenübungen unter 50 Prozent. Als \\ Therapeuten müssen wir diesem ernüchternden Ergebnis aber nicht hilflos gegenüberste- \\ hen. In Interviews geben Patienten klare Hinweise darauf, was sie in der Therapie benötigen, \\ um auf lange Sicht die Übungen auszuführen. Wir sollten auf sie hören.
}

Während der physiotherapeutischen Behandlung erarbeiten Sie mit Ihrem Patienten ein aktives Übungsprogramm und raten ihm, dieses Programm langfristig in einer bestimmten Dosierung durchzuführen. Sie empfehlen zum Beispiel Ihrem Patienten mit lumbalen Rückenschmerzen, wie in Guidelines empfohlen, langfristig ein Übungsprogramm zweimal in der Woche auszuführen [1]. Aber leider hoffen Sie als Therapeutin oft vergebens, dass der Patient Ihrem Rat folgt, denn Patienten vernachlässigen ihre Übungen nur allzu oft. Dabei hat die Wissenschaft den hohen Wert von „Exercise“ (aktive Bewegungsübungen) zur Verbesserung von Schmerz und Funktion bei muskuloskelettalen Beschwerden in vielen Studien aufgezeigt [2]. Sowohl für Rückenbeschwerden als auch für Arthroseschmerzen in der unteren Extremität ist der Einsatz von Selbstübungen inklusive heimbasierter Übungen langfristig sehr wirksam [3][4][5]. Evidenz gibt es also genug und trotzdem folgen die Patienten und Patientinnen dem Rat der Fachleute nicht immer im gewünschten Ausmaß.

\section{Adhärenz}

Wenn früher der Begriff Compliance, übersetzt Therapietreue oder (unreflektierte, unkritische) Folgsamkeit gegenüber ärztlichen Empfehlungen, verwendet wurde, empfiehlt die Weltgesundheitsorganisation WHO heute den Begriff Adhärenz, der folgendermaßen definiert wird [6]:

„The extent to which a person's behaviour - taking medication, following a diet, and/or executing lifestyle changes, corresponds with agreed recommendations from a health care provider."

„Das Ausmaß, in dem das Verhalten einer Person - die Einnahme von Medikamenten, die Befolgung einer Diät und/ oder die Durchführung von Lebensstiländerungen - mit den im Vorfeld mit einem Gesundheitsversorger vereinbarten Empfehlungen übereinstimmt.“
Diese Definition der WHO beinhaltet die kooperative Entwicklung der Therapie. Dies setzt voraus, dass Patient und Therapeut gemeinsam die Sichtweise des Patienten auf die Krankheit und die Notwendigkeit der Therapie in Betracht ziehen. Die Adhärenzforschung in der Medizin, die bisher vor allem die Medikamenteneinnahme analysierte, ermittelte eine durchschnittliche Adhärenz von zirka 50 Prozent. Das bedeutet, dass die Hälfte der Patienten die Einnahme nicht korrekt befolgen [6]. Mehrere Gründe für die NonAdhärenz konnten durch Studien ermittelt werden [7]:

- Unklarheiten des therapeutischen Vorgehens

- Barrieren seitens der Patienten

- Qualität der Arzt-Patienten Interaktion

- strukturelle Probleme der Betriebe

\section{Adhärenz im Kontext Selbstmanagement bei muskuloskelettalen Erkrankungen}

Im Kontext muskuloskelettaler Erkrankungen entspricht Adhärenz dem Ausmaß der Verhaltensveränderung, ein empfohlenes und vereinbartes Übungsprogramm auszuführen. In der Forschung und auch im Therapiealltag lässt sich die Adhärenz/Non-Adhärenz sehr schwer ermitteln. Um die Übungsadhärenz bei muskuloskelettalen Problemen zu messen, wurden in Studien Fragebogen, die Anzahl der tatsächlich wahrgenommenen Therapieeinheiten und oft auch die von Patienten angegebenen Übungseinheiten verwendet. Alle bisherig verwendeten Fragebogen haben jedoch psychometrische Unzulänglichkeiten bezüglich Validität und Reliabilität [8] und die Übungsfrequenz, häufig mit einem Logbuch dokumentiert, wird oft sehr uneinheitlich ermittelt und ausgewertet [9]. Die theoretischen Vorstellungen von Adhärenz lassen sich demnach nur sehr schwer auf Eigenübungen bei Patienten mit muskuloskelettalen Problemen in der Praxis umsetzen.

Die Adhärenz bei Eigenübungen wird in verschiedenen Arbeiten mit weniger als 50 Prozent angegeben [8]. Die Adhärenz/Non-Adhärenz steht aber mit dem Effekt der Behandlung in direktem Zusammenhang [10]. Für einen lang- 
fristigen Erfolg der Übungen ist somit eine gute Adhärenz eminent wichtig. Physiotherapeutinnen und Physiotherapeuten haben verschiedene Möglichkeiten, die Adhärenz mittels Patientenedukation zu verbessern. Die verschiedenen Maßnahmen, um die Selbsteffizienz der Patientinnen und Patienten in den einzelnen Behandlungsphasen zu unterstützen, wurden in dieser Zeitschrift von Prof. Dr. Karin Niedermann genau beschrieben [11]. Eine gelungene Patienten-Therapeuten Interaktion ist demnach die optimale Voraussetzung für eine gute Adhärenz. Weitere Vorgehensweisen zur Unterstützung der Adhärenz in der Behandlungsphase sind Motivationsprozesse, smarte Zielsetzungen und eine Aktionsplanung. Allerdings bildet die Erhaltungsphase nach den Behandlungseinheiten die kritische Zeit für eine langfristig erfolgreiche Adhärenz. Die Barrieren für diese wichtige Interventionszeit nach der eigentlichen Therapie sind vielfältig beschrieben [12], ihre direkten Einflüsse aber noch weitgehend ungeklärt.

\section{Die Phase nach der Therapie stellt den eigentlich kritischen Zeitraum für die Adhärenz dar.}

Dieser Artikel thematisiert die Patientenperspektive auf die Anwendung von Übungsprogrammen. Diese Ansichten könnten einen Beitrag dazu leisten, sowohl die Messverfahren der Adhärenz als auch die langfristige Übungsadhärenz bei muskuloskelettalen Problemen zu verbessern. Daraus resultieren Empfehlungen für die physiotherapeutische Behandlung.

\section{GELUNGENE INTERAKTION}

Während der Physiotherapiesitzungen erarbeiten die Physiotherapeutin und der Patient zwei Heimübungen. Sie beschließen gemeinsam, dass er diese Übungen täglich in zwei Serien zu zehn Wiederholungen ausführen wird. Der Patient plant, jeweils morgens vor der Arbeit zu üben. Nach eingehendem Clinical Reasoning spricht nichts gegen dieses Vorgehen. Das Verhalten des Patienten in den nächsten zwei Wochen zeigt eine gute Adhärenz, da er den Plan außer am Sonntagmorgen sehr gut in seinen Alltag integrieren kann.

\section{Positive Erfahrungen unterstützen}

In qualitativen Studien mit Patienten und Patientinnen mit Rückenschmerzen oder Hüft-/Kniearthrosebeschwerden wurden die Einflüsse auf die Übungsadhärenz evaluiert [5][13]. Eine positive oder negative Wirkung der Übungen scheint in vielen Fällen ausschlaggebend für die Adhärenz zu sein. Sammeln Patienten gute Erfahrungen mit den Übungen oder negative Erfahrungen, wenn sie nicht üben, beeinflusst dies verständlicherweise ihre Übungsadhärenz.

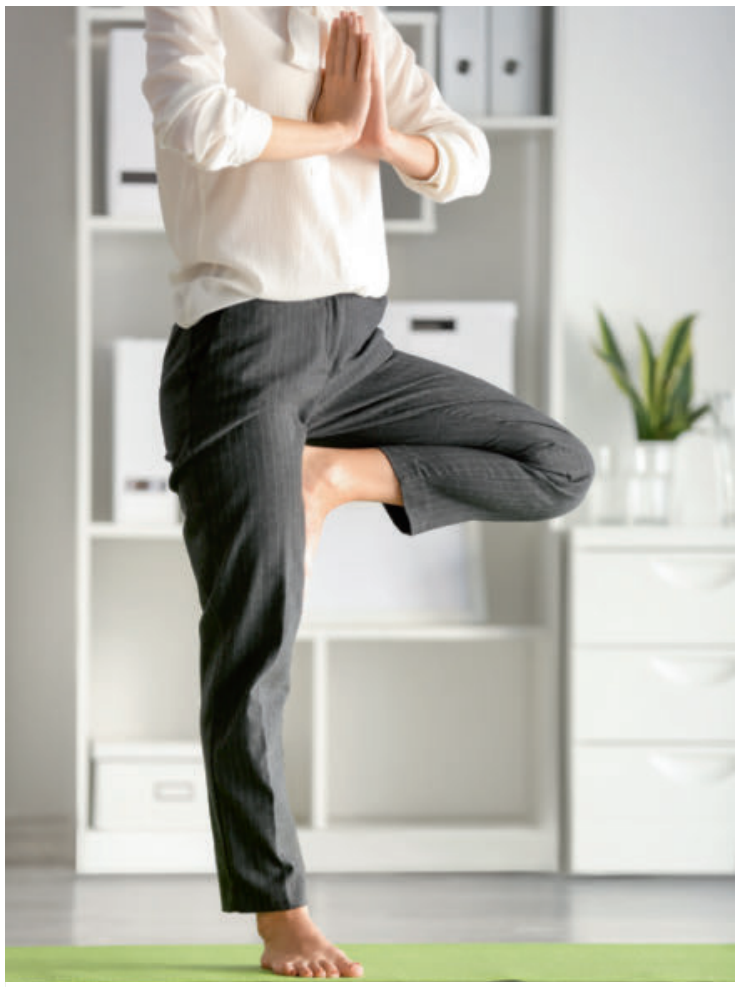

Abb. 1 Für die Umsetzung der Übung ist es wichtig, diese in den Alltag zu integrieren. (Symbolbild) (Quelle: Pixel-Shot/adobe.stock.com)

Ob sich die Schmerzen beim Üben verändern, steht dabei nicht immer im Vordergrund. Vielmehr nennen Patienten oft eine verbesserte Funktion oder Partizipation als positive Veränderung, ausgelöst durch das Übungsprogramm.

„....seit ich begonnen habe mit der Kräftigung der Muskulatur scheint mir, dass ich nicht mehr so häufig hinfalle. Dies ist sehr gut...es ist so beschämend. " (Patientin mit Hüftarthrose) [5]

Auch die Strategien zum Selbstmanagement werden durch möglichst positive Effekte und Erfahrungen unterstützt. Patienten berichten, dass es für die Umsetzung der Übungen wichtig ist, diese in ihren Alltag einzubinden ( $\triangleright$ Abb. 1). Dies kann sehr individuell ausfallen und hängt von den Lebensumständen und den Kapazitäten der Patienten ab. Sie erwähnen zum Beispiel unterschiedliche Übungsorte (Wohnung, Park, Wald, Fitnesscenter) oder Familiensituationen (Kinder, Enkel, Arbeitszeiten). Erstaunlicherweise sind diesbezüglich selbstgewählte und auch selbstorganisierte Umsetzungsstrategien für Patienten und Patientinnen sehr wichtig. Dies sollten die Therapeuten im Coaching, das heißt in einem begleiteten Prozess der Entscheidungsfindung, berücksichtigen.

„ICh mache meine rückenstabilisierenden Übungen im Anschluss an mein Fitnesstraining. “ [13]

„Ich kann die Übungen in meinen Sport (Nordic Walking) einbauen. “[6] 


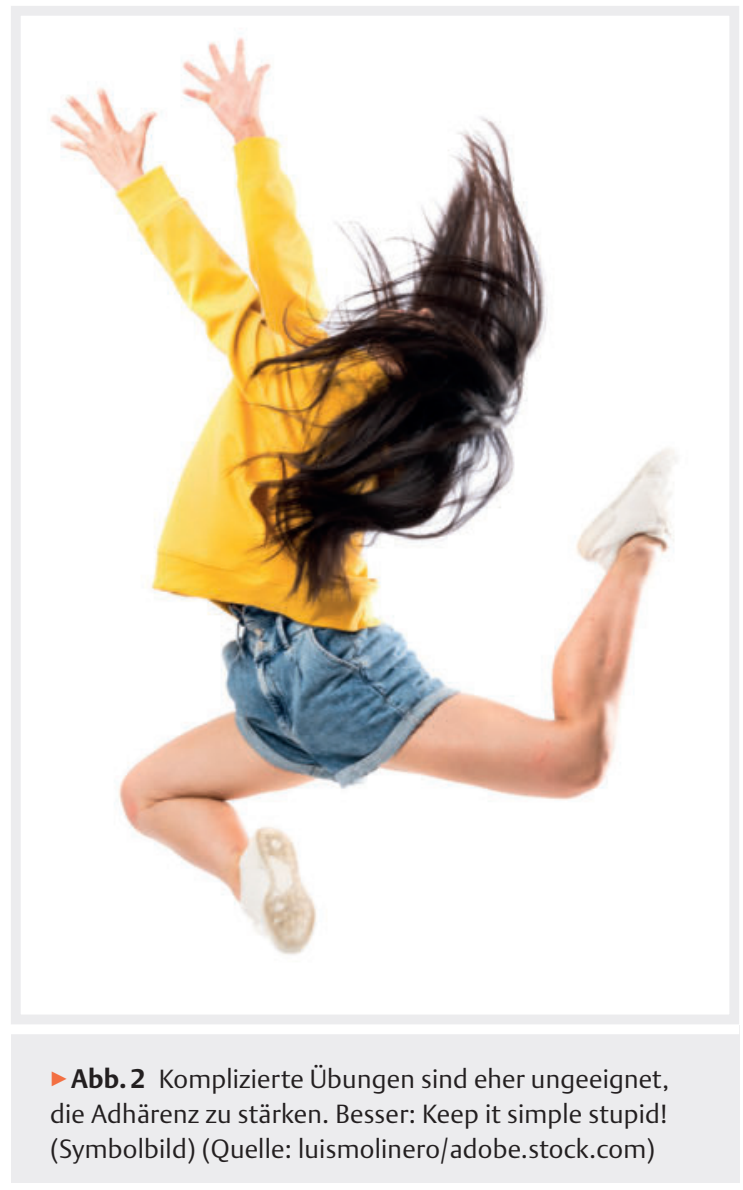

\section{Selbstvertrauen stärken}

Mehrere Reviews identifizierten eine fehlende Selbstmotivation und vorherige Non-Adhärenz-Erfahrungen als Barrieren [3][10][14]. Auch das mangelnde Selbstvertrauen, die Übungen korrekt durchzuführen, wurde als Hinderungsfaktor evaluiert [13]. Die Patienten bei den Übungen durch fachgerechte Instruktionen zu unterstützen, fördert daher die Adhärenz, was in vielen Studien belegt wurde [15]. Aus der Sicht der Patientinnen und Patienten ist die individuelle Anleitung in einer Physiotherapie wichtig. Patienten berichten, dass sie Angst haben, „etwas falsch zu machen“. Sie hätten die Übungen nicht mehr gemacht, weil sie sich „selber Schaden zufügen“ würden [16].

SYSTEM KISS - KEEP IT SIMPLE STUPID

Patienten wünschen sich einfach verständliche und leicht auszuführende Übungen, die trotzdem den nötigen Effekt erzielen.

Deshalb wünschen sich Patienten einfache und wenige Übungen. Übungen, die einfach zu lernen sind, können auch einfacher allein durchgeführt werden. Sie wünschen sich auch eine genaue Anleitung und Korrekturen, bis sie sich selbst zutrauen, die Übungen allein durchzuführen. Dadurch entwickeln sie ihr Selbstvertrauen. Selbstvertrau- en in die eigenen Fähigkeiten und Ressourcen sowie Unabhängigkeit von medizinischer Unterstützung sind persönliche Errungenschaften, die in direkten Zusammenhang mit guten Behandlungsresultaten gebracht werden können [17].

\section{Biopsychosoziale Einflüsse auf die Adhärenz}

Während in der Physiotherapie vor allem die biologischen Aspekte von muskuloskelettalen Problemen untersucht, analysiert und behandelt werden, steigt die Anerkennung der psychologischen und sozialen Anteile am Problem in der Gesamtbeurteilung [18]. Intrapersonelle Faktoren der Patienten und Patientinnen wie Denkweise, Einstellung, Motivation oder Erfahrung können als psychologische Barrieren auf das Selbstmanagement wirken. Bachmann et al. ermittelten in einem Review, dass auch eine schlechte soziale Unterstützung von Familie und Institutionen die Adhärenz zu heimbasierten Übungen wesentlich beeinflusst [3]. Gerade bei älteren Patienten haben diese Faktoren einen wesentlichen Einfluss, wie eine Befragung zum Thema Eigenengagement bei Hüftfrakturen ergab [19].

\section{Gerade bei älteren Patienten beeinflusst die soziale Unterstützung durch Familie oder Institutionen die Adhärenz wesentlich.}

Der Einsatz von verhaltenspsychologischen Verfahren verschiedenster Art kann die Adhärenz in Bezug auf Aktivität oder Exercise beeinflussen. Untersucht wurde zum Beispiel der Einfluss von Kommunikationsverfahren [20], Motivational Interviewing [21] oder der Control Theory [22]. All diesen Modellverfahren gemeinsam ist das Ziel, eine langfristige Verhaltensänderung herbeizuführen. Physiotherapeuten sollten die Patienten, die sich ihrer psychischen Eigenkapazitäten nicht sicher sind, unter sozialem Druck stehen oder mangelnde soziale Ressourcen haben, identifizieren, da diese eher Schwierigkeiten im Selbstmanagement zeigen [17]. Nur so können sie ihnen die nötige Unterstützung bieten.

\section{Langfristiger Support}

Während der regelmäßigen Besuche in der Physiotherapie wird die Voraussetzung für eine langfristige Exerciseadhärenz ermöglicht. Therapeut und Patient wählen in Zusammenarbeit die Übungen aus, die dann erlernt, wiederholt und den jeweiligen Bedürfnissen angepasst werden. Diese Therapeuten/Patienten Allianz ermöglicht individuelle Anpassungen.

Studien, die Teilnehmende über längere Zeit bei ihrem Exercise-Verhalten begleiten, haben den Vorteil, dass ein regelmäßiger Kontakt zu den Studienteilnehmern be- 
steht. Viele Teilnehmende haben diesen Faktor auch als positiv zur Förderung der Adhärenz bewertet [13]. Ein telefonischer Kontakt, das Führen und die Kontrolle eines Trainingstagebuches oder der Einsatz von elektronischen Feedbacktools könnten sehr unterstützend für eine langfristige Trainingsadhärenz sein [19][23] ( A Abb. 3). Um die langfristige Exercise-Adhärenz und damit das Selbstmanagement zu stärken, kann eine erneute Kontaktaufnahme von großer Bedeutung sein.

\section{Chancen der Physiotherapie}

Vorbei sind die Zeiten in denen der Physiotherapeut oder die Physiotherapeutin die Patienten nur „behandelt“. Es ist nicht nur das Expertenwissen der Therapeuten, das die Übungsstrategie bestimmt. Es ist nicht notwendig, dass Physiotherapeuten ihre Kenntnis von möglichst vielen, schwierigen Übungen unter Beweis stellen, zumal Topdown Ratschläge und Anweisungen oft eine schlechte Mitarbeit erzielen. Abkürzungen im Clinical Reasoning Prozess, indem aus Scham das Erfragen der psychosozialen Situation übergangen wird, führen öfters in die Sackgasse. Die gesteigerte Bedeutung der Rolle des Physiotherapeuten als Coach zur zielgerichteten, aktiven und langfristigen Exercise-Therapie der Patienten bietet Chancen für beide Seiten.

Die in Studien zu einer langfristigen Übungsadhärenz befragten Patientinnen und Patienten mit muskuloskelettalen Beschwerden wünschen sich in der Physiotherapie:

- positive Erfahrungen mit Übungen

- partizipative Auswahl der Heimübungen

- einfach verständliche, sicher umsetzbare und effiziente Übungsauswahl

- Anpassung der Anwendungsstrategie von Übungen an die jeweiligen Lebensumstände

- Begleitung der Übungsgewohnheiten, bis das nötige Selbstvertrauen zum Selbstmanagement erreicht ist

- Kenntnisnahme von persönlichen Barrieren für ein langfristiges Selbstmanagement

- keine Entlassung ohne Berücksichtigung von Eigenkapazitäten, Unsicherheit, Stress und Angst

- langfristige Nachkontrollen verschiedenster Art

- Einleiten von erweiterten Behandlungsmöglichkeiten, sofern erforderlich

\section{Fazit}

Die Perspektive der Patienten zum Adhärenzprozess kann uns wertvolle Hinweise auf verbesserte Erhebungsmöglichkeiten vermitteln. Noch sind die besten Messverfahren für die Adhärenz bei langfristigem Einsatz von Übungen nicht entwickelt. Neue digitale und technische Möglichkeiten könnten hierfür hilfreich sein. Im Therapiealltag sind das Patientenwissen, die Ressourcen der Patienten und die entsprechenden Fähigkeiten genau abzuwägen. Eine lang-

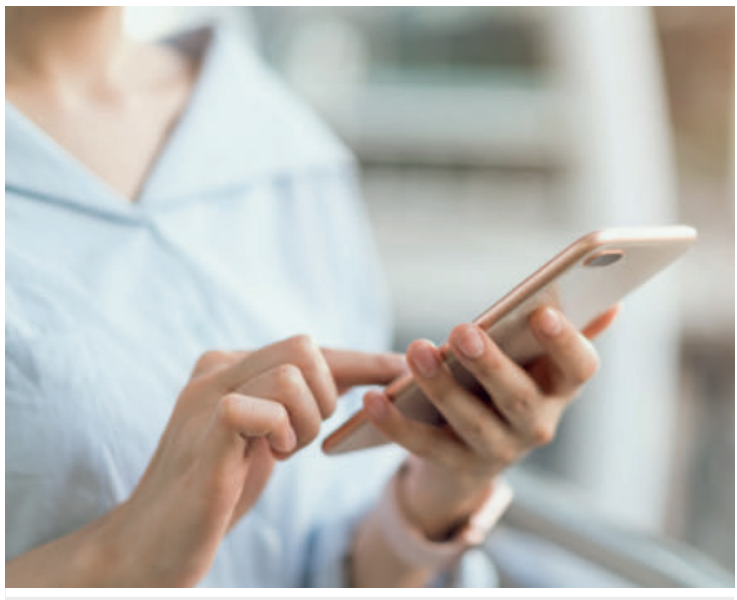

Abb.3 Telefonischer Kontakt nach der Therapie kann Patienten darin unterstützen, ihr Trainingsprogramm auf lange Sicht weiterzuverfolgen. (Symbolbild) (Quelle: sitthiphong/adobe.stock.com)

fristige Exercise-Adhärenz braucht auch ein langfristiges Coaching trotz Selbstmanagement.

\section{Autorinnen/Autoren}

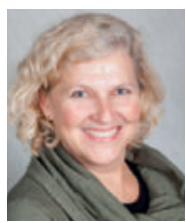

\section{Dr. Jeannette Saner}

PhD, Physiotherapeutin, ist Dozentin für muskuloskelettale Physiotherapie am Institut für Physiotherapie der Zürcher Hochschule für angewandte Wissenschaften in Winterthur, $\mathrm{CH}$. Sie beschäftigte sich in ihrer Dissertation an der Universität Maastricht, NL, mit aktiven Bewegungen bei nichtspezifischen Rückenschmerzen und dem Thema Adhärenz bei Heimprogrammen [24].

\section{Korrespondenzadresse}

seat@zhaw.ch

Literatur

Literaturverzeichnis am Ende der HTML-Version unter www. thieme-connect.de/products/manuelletherapie

\section{Bibliografie}

DOI https://doi.org/10.1055/a-1031-4342

manuelletherapie 2019; 23: 215-218

(c) Georg Thieme Verlag KG Stuttgart · New York ISSN 1433-2671 